\title{
NOUVELLE
}

\section{Régulation de la transcription par le coactivateur TFIID}

Gabor Papai, Patrick Schultz
Département de biologie structurale intégrée,

Institut de génétique et de biologie moléculaire et cellulaire, U964 Inserm, UMR7104 CNRS/Université de Strasbourg,

1, rue Laurent Fries, 67404 IIIkirch, France.

patrick.schultz@igbmc.fr
> La transcription des gènes codant pour des protéines est précisément régulée afin de permettre l'adaptation des cellules eucaryotes à une multitude de signaux internes ou externes. L'expression des gènes requiert la formation d'un complexe de pré-initiation (PIC) sur une séquence d'ADN promotrice placée en amont de la région codante [1]. Sept facteurs de transcription généraux sont nécessaires et suffisants pour former le PIC et enclencher la transcription in vitro lorsque le promoteur contient une séquence TATA. Le facteur TBP (TATA box binding protein) interagit spécifiquement avec la séquence TATA, le facteur TFIIA stabilise cette interaction alors que TFIIB interagit avec le complexe TBP-ADN et recrute I'ARN polymérase II. TFIIF, TFIIE et TFIIH interagissent avec l'enzyme et assurent sa conversion en une forme compétente pour l'élongation de la transcription. Ce schéma de base n'explique pas toutes les propriétés du mécanisme de transcription. Le séquençage du génome humain a montré que les promoteurs dans leur majorité ne possèdent pas de séquence TATA [2]. Par conséquent la protéine TBP ne peut pas, à elle seule, reconnaître le promoteur, et d'autres fonctions de reconnaissance de I'ADN sont requises. Par ailleurs, TBP n'existe pas sous une forme libre dans la cellule mais s'associe à d'autres protéines pour former des complexes multiprotéiques de taille parfois supérieure à $1 \mathrm{MDa}[1]$. Enfin, le système des sept facteurs généraux ne répond pas aux activateurs de transcription qui sont les derniers maillons des voies de signalisation cellulaires. Les activateurs stimulent la transcription des gènes en se liant de manière spécifique à des séquences d'ADN placées entre quelques centaines à plusieurs milliers de paires de bases en amont du promoteur. La nécessité d'établir une interaction physique entre les facteurs généraux et les activateurs de transcription a conduit à la notion de coactivateur transcriptionnel.

\section{Le coactivateur TFIID}

Le facteur de transcription TFIID est I'un de ces coactivateurs et contient TBP ainsi que 14 sous-unités nommées TAF pour TBP associated proteins [3]. La structure atomique de TBP montre que cette protéine adopte une forme de croissant et interagit avec la boîte TATA par l'intermédiaire de sa face concave [4]. Mais d'autres TAF, tel que TAF2, participent à la reconnaissance du promoteur et, dans certains cas, rendent facultative la présence d'une boîte TATA [3]. Plusieurs TAF, dont TAF4 et TAF12, établissent des liaisons avec des activateurs transcriptionnels et contribuent au recrutement de TFIID à proximité des gènes à transcrire. La sous-unité TAFl contient dans sa partie aminoterminale une séquence dénommée TAND (TAF N-terminal domain) qui mime la double hélice d'ADN et interagit avec la face concave de TBP pour empêcher sa liaison à l'ADN [5]. Cette fonction auto-inhibitrice empêche TBP de se lier sans discrimination sur n'importe quelle séquence TATA au risque d'enclencher la transcription de manière incontrôlée. Les activateurs de transcription doivent jouer un rôle essentiel pour lever cette inhibition et permettre l'interaction de TBP avec les promoteurs situés à proximité de leurs sites de liaison. Les étu- des structurales récentes ont permis un éclairage nouveau sur ce phénomène mal compris.

\section{Analyse structurale}

Les premiers modèles 3-D de TFIID ont été obtenus par microscopie électronique et ont mis en évidence une organisation modulaire (Figure 1A) [6]. L'utilisation d'anticorps a permis de positionner les différents TAF dans la structure de TFIID [7]. Les positions de TBP et de TAF2, tous deux impliqués dans la liaison avec le promoteur, ainsi que celle de l'extrémité amino-terminale de TAFl, qui comprend les motifs inhibiteurs TAND, ont été déterminées (Figure IA). Afin de mieux comprendre le rôle des activateurs de transcription, nous avons analysé par microscopie électronique des complexes formés entre TFIID et l'activateur Rapl de la levure S. cerevisiae [8]. Nous avons identifié le site d'interaction de Rapl sur TFIID à proximité des sous-unités TAFl2 et TAF4 qui sont les partenaires d'interaction in vitro de Rapl (Figure IA). TFIID ne peut enclencher la transcription et les activateurs ne peuvent exercer leur fonction qu'en présence de TFIIA qui contribue à lever l'auto-inhibition de TBP par les séquences TAND. L'interaction de TFIID avec TFIIA, en présence de I'ADN promoteur, a également été caractérisée par microscopie électronique [8] et il apparaît que TFIIA interagit à proximité de TBP (Figure 1A). Afin de comprendre comment TFIIA communique avec les activateurs pour stimuler la transcription, un complexe entre TFIIA, TFIID, Rapl et I'ADN promoteur a été analysé [8]. L'analyse structurale a révélé que l'activateur Rapl interagit directement avec 


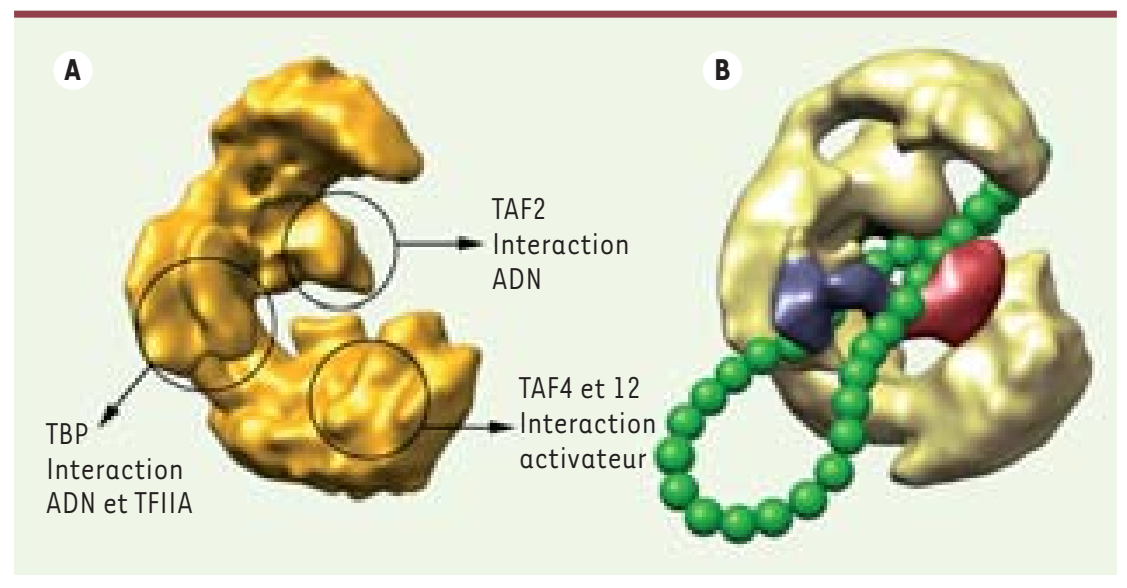

TFIIA et provoque un changement de conformation important de TFIIA qui fait une rotation de plus de $110^{\circ}$ (Figure IB). Comme TFIIA contacte directement TBP, il est vraisemblable que la position de TBP soit également modifiée par la liaison avec l'activateur et que ce changement de conformation de TBP contribue à lever l'auto-inhibition due aux séquences TAND de TAFl et favorise la transition vers un complexe d'initiation de la transcription. Les observations en microscopie électronique ont également montré l'existence d'une boucle d'ADN (Figure 1B) dont la longueur s'adapte à la grande variété de distances entre le site de liaison de l'activateur et le site d'initiation.

Ces résultats montrent que les coactivateurs agissent comme des machines moléculaires qui, par le jeu de transitions structurales successives, favorisent l'évolution d'un système métastable.
Quels avantages les eucaryotes ont-ils pu trouver à instaurer les coactivateurs alors que les procaryotes ont privilégié une interaction directe entre I'ARN polymérase et les activateurs? La notion de régulation croisée permettant l'intervention de plusieurs activateurs sur le promoteur d'un même gène est à cet égard très attrayante. Des expériences biochimiques et structurales ont montré que plusieurs activateurs peuvent se lier en même temps et sur des sites distincts sur TFIID [9]. Ces interactions permettent de combiner plusieurs voies de signalisation et augmentent la diversité des réponses dans un système intégrant différents activateurs. $\diamond$

\section{Transcriptional regulation}

by the coactivator TFIID

\section{CONFLIT D'INTÉRÊTS}

Les auteurs déclarent n'avoir aucun conflit d'intérêts concernant les données publiées dans cet article.
Figure 1. Rôle de TFIID dans l'activation de la transcription. A. Structure tridimensionnelle du facteur TFIID obtenu par microscopie électronique et localisation des sous-unités impliquées dans les interactions avec l'ADN et les facteurs de transcription. B. Formation d'une interaction entre l'activateur Rapl (rouge) et le facteur TFIIA (bleu) dans un complexe quaternaire entre TFIID, TFIIA, Rapl et le promoteur. Le chemin de I'ADN est représenté schématiquement en vert.

\section{RÉFÉRENCES}

1. Burley SK, Roeder RG. Biochemistry and structural biology of transcription factor IID. Annu Rev Biochem $1996 ; 65: 769-99$.

2. Sandelin A, Carninci P, Lenhard B, et al. Mammalian RNA polymerase II core promoters: insights from genome-wide studies. Nat Rev Genet 2007 ; 8 : 424-36.

3. Cler $\varepsilon$, Papai G, Schultz P, Davidson I. Recent advances in understanding the structure and function of general transcription factor TFIID. Cell Mol Life Sci 2009; $66: 2123-34$

4. Burley SK. The TATA box binding protein. Curr Opin Struct Biol $1996 ; 6: 69-75$.

5. Kokubo T, Yamashita S, Horikoshi M, et al. Interaction between the $\mathrm{N}$-terminal domain of the $230-\mathrm{kDa}$ subunit and the TATA box-binding subunit of TFIID negatively regulates TATA-box binding. Proc Natl Acad Sci USA $1994 ; 91$ : 3520-4.

6. Brand M, Leurent C, Mallouh V, et al. Three dimensional structures of the TAF ${ }_{\|}$-containing complexes TFIID and TFTC. Science $1999 ; 286$ : 2151-3.

7. Leurent C, Sanders S, Ruhlmann C, et al. Mapping histone fold TAFs within yeast TFIID. EMBO 2002 $21: 3424-33$.

8. Papai G, Tripathi MK, Ruhlmann C, et al. TFIIA and the transactivator Rapl cooperate to commit TFIID for transcription initiation. Nature $2010 ; 465$ : 956-60.

9. Liu WL, Coleman RA, Ma $\varepsilon$, et al. Structures of three distinct activator-TFIID complexes. Genes Dev 2009; $23: 1510-21$.

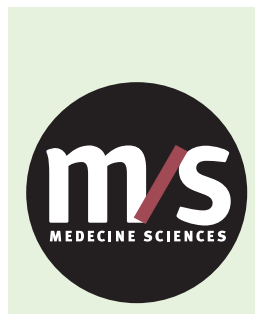

Tarifs d'abonnement M/S - 2010

$>$ Grâce à $m / s$, vivez en direct les progrès des sciences biologiques et médicales

Abonnez-vous

à Médecine/Sciences
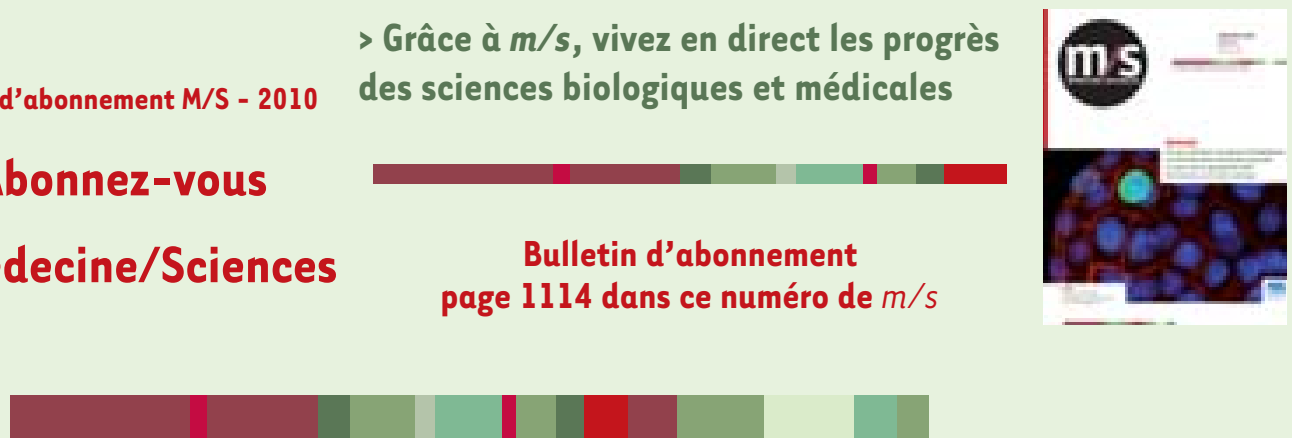